\title{
Prevalencia de marcadores serológicos ANCA y ASCA en una población con colitis ulcerosa
}

\author{
Teresa Vergara $A^{1,5}$, Pamela Cofré $L^{1}$, Soledad Cifuentes $A^{2}$, \\ Ursula Pulgar $A^{2}$, Claudio Puebla $A^{3}$, Susana Velasco $P^{4}$. \\ Presence of antineutrophil \\ cytoplasmic antibodies (ANCA) \\ and anti Saccharomyces cerevisiae \\ antibodies (ASCA) among patients \\ with ulcerative colitis
}

Background: The diagnosis of inflammatory bowel disease is supported by clinical findings and complementary tests. The presence of specific serological markers could be helpful in the characterization of this condition. Aim: To assess the prevalence of ANCA and ASCA in a group of patients with ulcerative colitis (UC) and its association with clinical features. Material and Methods: Sixty four patients with UC in remission (age range 16-72 years, 33 males) were studied. In a venous blood sample ANCA were measured by indirect immunofluorescence and ASCA by enzyme immune assays for IgG and IgA. Results: Forty four percent of patients were positive for ANCA, $9 \%$ for ASCA and $6 \%$ for both markers. There was a significant correlation between the presence of ANCA and duration of the UC ( $<5$ years $50 \%, 5-10$ years $42.9 \%, 15$ years $30 \%$ ) and the number of crises (one crises $31 \%, 2-5$ crises $51.9 \%$ and $>5$ crises 87.5 ). The proportion of colectomized patients with positive ANCA was higher (57.1\%). Conclusions: The prevalence of ANCA in the studied population is similar to the published data. The presence of ANCA was significantly higher in UC patients with shorter evolution, higher number of crises and in those with a history of colectomy. There was a low prevalence of ASCA positive patients (Rev Méd Chile 2006; 134: 960-4).

(Key words: Antibodies, antineutrophil cytoplasmic; Colitis, ulcerative; Saccharomyces cerevisiae)

Recibido el 29 de agosto, 2005. Aceptado el 16 de enero, 2006.

${ }^{1}$ Departamento de Gastroenterología, Hospital Naval Almirante Nef. ${ }^{2}$ Programa de Formación de Especialista en Medicina Interna, Universidad de Valparaíso. ${ }^{3}$ Servicio de Medicina y ${ }^{4}$ Consultorio Médico Naval, Hospital Naval Almirante Nef. ${ }^{5}$ Centro Diagnóstico Digestivo (CEDID). Viña del Mar.

$\mathrm{N}$ o existe síntoma ni signo alguno patognomónico para diagnosticar las enfermedades inflamatorias intestinales crónicas (EII) tales como colitis ulcerosa (CU) y enfermedad de Crohn (EC). Para el diagnóstico diferencial de estas dos entida-

Correspondencia a: Dra. María Teresa Vergara Albarracín. Errázuriz 634, Viña del Mar. Fax: 32284621-2684284.

E-mail: vergaraalbarracín@hotmail.com. des, se utiliza una combinación de datos clínicos, radiológicos, endoscópicos e histológicos. Sin embargo, cuando existe compromiso del colon, el diagnóstico diferencial entre colitis ulcerosa y enfermedad de Crohn puede ser difícil, quedando un subgrupo de pacientes (10 a 15\%) clasificados como portadores de colitis indeterminada ${ }^{1,2}$.

En las EII se han descrito alteraciones inmunológicas, tanto sistémicas como localizadas en el 
propio tracto intestinal. En 1990, Saxon et $\mathrm{al}^{3}$ encontraron la presencia de anticuerpos anticitoplasma de los neutrófilos (ANCA) en pacientes con EII. Desde entonces, estudios posteriores han confirmado dicha asociación ${ }^{3-13}$. Según su patrón de inmunofluorescencia indirecta sobre leucocitos polimorfonucleares (PMN) humanos fijados con etanol se ha descrito: el patrón citoplasmático granular difuso cANCA, causado por anticuerpos antiproteinasa 3 (PR3), el patrón de tinción citoplasmático perinuclear pANCA, causado por anticuerpos dirigidos en contra de diferentes antígenos, entre ellos: mieloperoxidasa, elastasa, cathepsina G, azurocidina, lactoferrina, lisosima, etc. y un tercer grupo «atípico», del cual no se conoce la especificidad antigénica y que presenta un patrón de tinción citoplasmática difusa junto con tinción perinuclear, sin embargo, en la literatura el ANCA asociado con EII ha recibido históricamente el nombre de $p$-ANCA $\Downarrow^{4-19}$.

En estos pacientes se ha detectado también otro tipo de anticuerpos, dirigidos contra una levadura, los anti-Saccharomyces Cerevisiae (ASCA), que pueden ser de tipo IgG o IgA y que se identifican preferentemente en los pacientes con EC.

No existe evidencia científica que sugiera la hipótesis de que los ANCA o los ASCA desempeñen un papel patogénico en el desarrollo de la CU o la EC, respectivamente, más bien, su presencia parece reflejar una alteración en la regulación inmunológica que subyace en las EII, originada por la exposición a un antígeno en sujetos genéticamente predispuestos. La investigación de estos marcadores se ha realizado fundamentalmente en países europeos y de Norteamérica, desconociéndose lo que ocurre con éstos en nuestro medio.

El objetivo de este trabajo fue determinar la prevalencia de los marcadores ANCA y ASCA en un grupo de pacientes portadores de CU y establecer si su presencia se relaciona con ciertas características clínicas y pronósticas de esta enfermedad.

\section{Material y MÉTOdO}

Previo a consentimiento informado y aprobación del protocolo por el Comité de Etica del Hospital Naval Almirante Nef de Viña del Mar, se revisaron las historias clínicas de 64 pacientes con diagnóstico clínico e histológico de CU en remisión, contro- lados en el Hospital Naval Almirante Nef y Centro Diagnóstico Digestivo (CEDID) de Viña del Mar.

La CU fue diagnosticada según criterios clínicos, endoscópicos e histopatológicos. Se recolectó, de las historias clínicas, información sobre: edad, sexo, años de evolución, extensión, número de crisis, tratamiento de mantención, necesidad de terapia corticoesteroidal, inmunosupresora o ambas, compromiso extradigestivo y antecedente de colectomía.

Los ANCA se evaluaron por técnica de inmunofluorescencia indirecta (IFI), utilizando como sustratos células Hep-2 y leucocitos polimorfonuclerares humanos. Para su adecuada caracterización, se emplearon placas de PMN fijados con etanol y formalina. Los ASCA se evaluaron por técnica ELISA (IgG e IgA). Todos los reactivos se obtuvieron de INOVA Diagnostics, Inc. San Diego CA, USA.

Los valores de ANCA se expresaron en relación a su dilución (titulación), considerando como valores positivos aquellos iguales o mayores a 1:40.

Los valores de ASCA se expresaron en unidades internacionales por ml. Se consideró como valores de referencia para ASCA IgG e IgA: entre 0,0 y 20,0 UI: negativo, entre 20,1 y 24,9 UI limítrofe y valores mayores a 25 UI positivos.

Los resultados fueron analizados con método estadístico de tipo descriptivo, usando promedios y porcentajes. Las asociaciones se evaluaron por medio de Chi cuadrado.

\section{RESULTADOS}

Sesenta y cuatro pacientes con CU fueron incluidos en el estudio. La edad promedio fue de 43,1 años con un rango entre 16 y 72 años. Cincuenta y un por ciento fueron hombres. Según el tiempo de evolución de la enfermedad, la distribución de los pacientes fue: menos de 5 años, 53,1\%, entre 5 y 10 años, 20,3\%, entre 10 y 15 años, 10,9\% y mayor de 15 años, 15,6\%. Según la extensión de la enfermedad, se encontró que $25 \%$ comprometía solamente el recto, $46,9 \%$ presentaba rectosigmoiditis, 9,4\% colitis izquierda y $18,8 \%$ de los pacientes tenía pancolitis. Dentro del grupo, siete pacientes eran colectomizados.

Treinta y nueve pacientes habían tenido tratamiento con glucocorticoides orales y 20 pacientes recibían Azatioprina al momento de entrar al protocolo. Todos los pacientes recibían, además, derivados de ácido 5-aminosalicílico. 
Con respecto a la serología del grupo, ésta fue positiva en $46,9 \%$ (30) de los pacientes con un intervalo de confianza (IC) $95 \%(34,3-59,8)$. De éstos, resultaron ANCA (+) 37,5\% (24), ASCA (+) $3,1 \%$ (2) y ambos marcadores positivos 6,3\% (4).

Según el patrón de distribución de la inmunofluorescencia, 25\% (6) de los pacientes ANCA (+) presentó un patrón perinuclear (pANCA), y $75 \%$ (18) un patrón atípico.

Se encontró diferencia significativa $(p<0,05)$ al relacionar la presencia de ANCA con el tiempo de evolución de la CU. En colitis de menos de 5 años, $50 \%$ de los pacientes tenía serología positiva, entre 5 y 10 años, 53,8\%, entre 10 y 15 años, 42,9\% y mayor de 15 años $30 \%$ (Figura 1). También hubo relación entre el número de crisis de colitis ulcerativa y el porcentaje de pacientes con ANCA (+): Pacientes con una crisis presentaron serología ANCA positiva, en $31 \%$ de los casos, pacientes con 2 a 5 crisis, presentaron ANCA (+) en 51,9\% y aquellos con más de 5 crisis, 87,5\% (Figura 2).

Los pacientes colectomizados presentaron ANCA ( + ) en $57,1 \%$ y los no colectomizados en $45,6 \%(\mathrm{p}<0,05)$ (Figura 3).

Sólo seis pacientes tuvieron serología positiva para ASCA, y se observó una tendencia de mayor prevalencia del marcador en los pacientes con compromiso rectosigmoideo, a mayor número de crisis y en colectomizados.

Con respecto a manifestaciones extradigestivas, éstas se presentaron en 15 pacientes: 1 hepatitis autoinmune, 1 pioderma gangrenoso, 3 sacroileítis, 8 con artralgias, 2 con manifestaciones oculares de los cuales uno presentó además eritema nodoso. Del grupo total 40\% (6 pacientes) presentaron serología positiva, lo que no difiere significativamente de los pacientes sin manifestaciones extradigestivas (49\%). Al revisar en forma dirigida aquellos pacientes con manifestaciones articulares, se observó una prevalencia de serología positiva de $36,4 \%$ (4/11 pacientes), lo cual tampoco se diferenció significativamente del grupo global de CU.

No se encontró diferencias al relacionar la serología con la edad, aunque hubo una tendencia a presencia menor de marcadores a mayor edad. Tampoco hubo diferencia al relacionar la serología con el sexo, extensión de la enfermedad, uso de corticoides o inmunomoduladores.

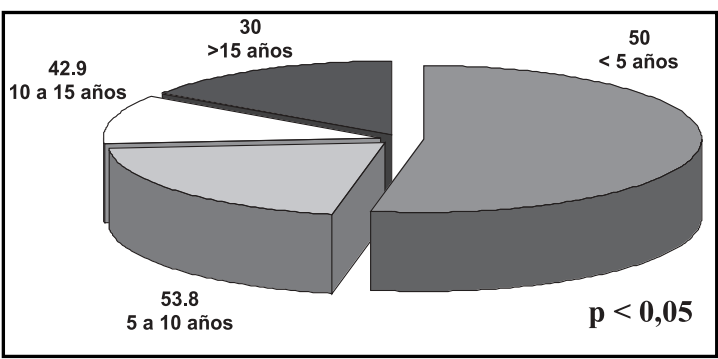

Figura 1. Porcentaje de pacientes con ANCA según tiempo de evolución de la enfermedad.

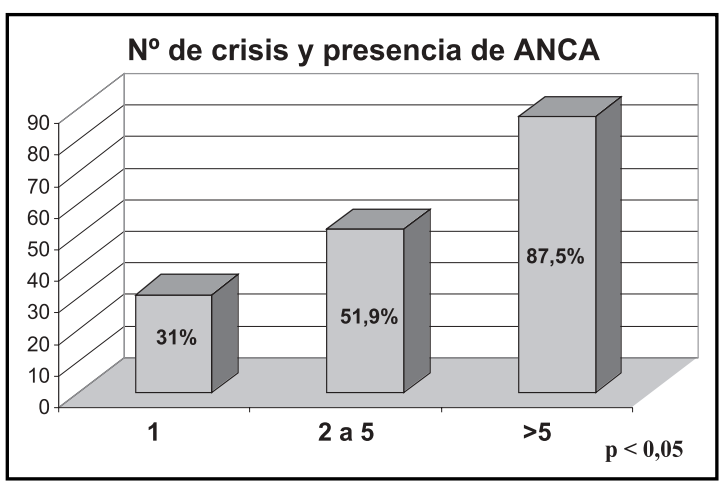

Figura 2. Relación entre el número de crisis y frecuencia de la presencia de ANCA.

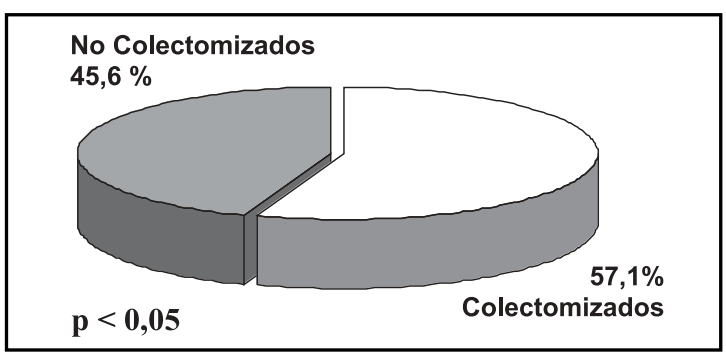

Figura 3. Distribución de pacientes con ANCA según antecedente de cirugía.

\section{DisCUSIÓN}

La relación entre la presencia de ANCA y la CU aún sigue siendo controversial-5,13,20-22. La utilidad de la determinación de este marcador para diferenciar entre los pacientes con CU y los controles sanos carece de interés en la práctica diaria. Mucho más interesante para el clínico, son los estudios que evalúan la exactitud de los ANCA para el diagnóstico de la CU entre los pacientes con clínica compatible. A partir de los datos de la 
literatura, se concluye que los ANCA tienen una sensibilidad subóptima (media, 58\%), sin embargo la especificidad es considerablemente elevada (media, 93\%). La presencia de uno u otro tipo de anticuerpos podría tener implicaciones pronósticas y terapéuticas; así, permite al clínico orientarse, en un cuadro de crisis inflamatoria sin elementos claros para definir si se trata de una colitis ulcerosa o enfermedad de Crohn. La diferenciación entre ambas enfermedades es especialmente relevante a la hora de tomar determinadas conductas terapéuticas ${ }^{2}$. Pese a que la mayoría de los estudios han demostrado que la presencia de ANCA o su titulación no guardan relación con la actividad de la CU, en nuestra población encontramos una relación estadísticamente significativa entre mayor número de crisis y la presencia del marcador. El hallazgo de una mayor prevalencia de ANCA en pacientes colectomizados, nos permite plantear que su presencia podría ser un marcador de peor pronóstico. La presencia de ANCA fue significativamente mayor en nuestros pacientes con menor tiempo de evolución de la enfermedad. Una probable explicación podría ser el efecto del tratamiento inmunosupresor sobre el marcador, lo que permitiría aventurar que la medición de ANCA podría tener su utilidad máxima en el diagnóstico diferencial al inicio de la enfermedad. Sin embargo, esta aseveración deberá evaluarse en el futuro con diseños prospectivos para su correcta interpretación. Nuestros resultados confirman lo encontrado en otros centros en cuanto a la baja prevalencia de ASCA en pacientes con CU. Su ausencia, junto con la presencia de serología positiva para ANCA en pacientes con EII nos orienta al diagnóstico de CU. Por el hecho de

\section{REFERENCIAS}

1. Stewentus J, Adnerhill I, Ekelund G, Floren CH, Fork FT, JANZON L ET AL. Ulcerative colitis and indeterminate colitis in the city of Malmo, Sweden. A 25-year incidence study. Scand J Gastroenterol 1995; 30: 38-43.

2. Gisbert JP, Gomolón F, Maté J, Pajares JM. The role of anti-neutrophil cytoplasmic antibodies (ANCA) and anti-Saccharomyces cerevisiae antibodies (ASCA) in inflammatory bowel disease. Gastroenterol Hepatol 2003; 26: 312-24. encontrarse el marcador en pacientes con mayor número de crisis y colectomizados, nos permite aventurar que podría tratarse de un subgrupo de pacientes con enfermedad de curso más agresiva, sin embargo, esta tendencia deberá confirmarse aumentando el número de los pacientes a estudiar. En nuestro trabajo sólo se pudo determinar la sensibilidad que corresponde a los pacientes con serología positiva. No siendo posible calcular el valor predictivo positivo (VPP) y negativo (VPN), como tampoco especificidad, dado que el estudio solamente se realizó en pacientes con CU. Para dichos cálculos se requiere de un grupo control que no tenga la enfermedad y que por problemas de costo de los reactivos no se pudo incorporar.

Como se ha comentado en la literatura, es decepcionante la sensibilidad de estos marcadores serológicos, lo que limita en forma considerable su utilidad como método de cribado de la población con sospecha de EII, si bien su elevada especificidad apoya con relativa seguridad el diagnóstico de EC o de CU cuando el resultado es positivo. La determinación simultánea de ambos anticuerpos, pANCA y ASCA, tiene una cierta utilidad para diferenciar entre CU y EC, pero siempre que el resultado de esta combinación incluya la positividad de un anticuerpo, a la vez que la negatividad del otro.

Estos marcadores no disponen de un VPN suficientemente elevado como para eliminar la necesidad de realizar otras pruebas diagnósticas en los pacientes con la sospecha de $\mathrm{EII}^{2}$. Sin embargo, creemos que pueden utilizarse como una herramienta más, dentro del estudio de estos pacientes, y realizarse precozmente cuando se sospeche el diagnóstico de EII.

3. Saxon A, Shanahan F, Landers C, Ganz T, Targan S. A distinct subset of antineutrophil cytoplasmic antibodies is associated with inflammatory bowel disease. J Allergy Clin Immunol 1990; 86: 202-10.

4. Lombardi G, Annese V, Piepol A, Bovio P, Latiano A, Napoltano G et al. Antineutrophil cytoplasmic antibodies in inflammatory bowel disease: clinical role and review of the literature. Dis Colon Rectum 2000; 43: 999-1007.

5. Duerr RH, Targan SR, Landers CJ, Sutherland LR, SHANAHAN F. Antineutrophil cytoplasmic antibodies in ulcerative colitis: comparison with other coliti- 
des/diarrheal illnesses. Gastroenterology 1991; 100: 1590-6.

6. Duerr RH, Targan SR, Landers CJ, Larusso NF, LINDSAY KL, WIESNER RH ET AL. Neutrophil cytoplasmic antibodies: a link between primary sclerosing cholangitis and ulcerative colitis. Gastroenterology 1991; 100: 1385-91.

7. Seibold F, Weber P, Kifin R, Berg PA, Wiedmann KH. Clinical significance of antibodies against neutrophils in patients with inflammatory bowel disease and primary sclerosing cholangitis. Gut 1992; 33: 657-62.

8. Broekroelofs J, Mulder AHL, Nelis GF, Westerveld BD, Cohen Tervaert JW, Kallenberg CGM. Antineutrophil cytoplasmic antibodies (ANCA) in sera from patients with inflammatory bowel disease (IBD). Dig Dis Sci 1994; 39: 545-9.

9. Vasilauskas EA, Plevy SE, Landers CJ, Binder SW, Ferguson DM, Yang H et al. Perinuclear antineutrophil cytoplasmic antibodies in patients with Crohn's disease define a clinical subgroup. Gastroenterology 1996; 110: 1810-9.

10. Oudkerk Pool M, Euerbroek PM, Ridwan BU, GoidsChmeding R, Von Blomberg BME, Peña AS et al. Serum antineutrophil cytoplasmic autoantibodies in inflammatory bowel disease are mainly associated with ulcerative colitis. A correlation study between perinuclear antineutrophil cytoplasmic autoantibodies and clinical parameters, medical, and surgical treatment. Gut 1993; 34: 46-50.

11. Seibold F, Slametschka D, Gregor M, Weber P. Neutrophil autoantibodies: a genetic marker in primary sclerosing cholangitis and ulcerative colitis. Gastroenterology 1994; 107: 532-6.

12. Kiein R, Eisenburg J, Weber P, Seibold F, Berg PA. Significance and specificity of antibodies to neutrophils detected by western blotting for the serological diagnosis of primary sclerosing cholangitis. Hepatology 1991; 14: 1147-52.

13. Papo M, Quer JC, Pastor RM, García-Pardo G, Olona M, Prats E et al. Antineutrophil cytoplasmic autoantibodies in inflammatory bowel disease. Med Clin (Barc) 1998; 110: 11-5.

14. Hoffman G, Specks U. Antineutrophil Cytoplasmic Antibodies. Arthritis \& Rheumatism 1998;41:1521-37.
15. Rump JA, Scholmerich J, Gross V, Roth M, HeifesrieDER R, RAUTMANN A ET AL. A new type of perinuclear anti-neutrophil cytoplasmic antibody (p-ANCA) in active ulcerative colitis but not in Crohn's disease. Immunobiology 1990; 181: 406-13.

16. Reumaux D, Sendid B, Poulain D, Duthileul P, Dewit O, Colombel JF. Serological markers in inflammatory bowel diseases. Best Pract Res Clin Gastroenterol 2003; 17: 19-35.

17. Linskens R, MaLiant-Hent RC, Groothuismink ZM, Bakker-Jonges LE, Van De Merwe JP, HooijKaAs H et AL. Evaluation of serological markers to differentiate between ulcerative colitis and Crohn's disease: pANCA, ASCA and agglutinating antibodies to anaerobic coccoid rods. Eur J Gastroenterol Hepatol 2002; 14: 1013-8.

18. Quinton JF, Sendid B, Reumaux D, Duthileul P, Cortot A, Grandbastien B et al. Anti-Saccharomyces cerevisiae mannan antibodies combined with antineutrophil cytoplasmic autoantibodies in inflammatory bowel disease: prevalence and diagnostic role. Gut 1998; 42: 788-91.

19. Peeters $M$, Joossens S, Vermeire S, Vuetinck $R$, BossuYt X, RUtGeerts P. Diagnostic value of antiSaccharomyces cerevisiae and antineutrophil cytoplasmic autoantibodies in inflammatory bowel disease. Am J Gastroenterol 2001; 96: 730-4.

20. Sandborn WJ, Landers CJ, TREMaine WJ, TaRgan SR. Association of antineutrophil cytoplasmic antibodies with resistance to treatment of left-sided ulcerative colitis: results of a pilot study. Mayo Clin Proc 1996; 71: 431-6.

21. Sobajima Ozaki S, Okazaki T, Osakada F, Sumita $\mathrm{S}$, Mori K, NaKaO K. Anti-neutrophil cytoplasmic antibodies (ANCA) in ulcerative colitis: anti-cathepsin $\mathrm{G}$ and a novel antibody correlate with a refractory type. Anti-neutrophil cytoplasmic antibodies (ANCA) in ulcerative colitis: anti-cathepsin $\mathrm{G}$ and a novel antibody correlate with a refractory type. Clin Exp Immunol 1996; 105: 120-4.

22. Brimnes J, Neilsen OH, Wiik A, HeegaArd NH. Autoantibodies to molecular targets in neutrophils in patients with ulcerative colitis. Dig Dis Sci 1999; 44: 415-23.

\section{Agradecimientos}

Este trabajo contó con el apoyo del QF. Sr. Marcelo Tapia Carrere, del Laboratorio Clínico ACLIN de Viña del Mar. 